\title{
BMJ Open Electrosclerotherapy for capillary malformations: study protocol for a randomised within-patient controlled pilot trial
}

\author{
Sophie E R Horbach, ${ }^{1}$ Albert Wolkerstorfer, ${ }^{2}$ Daniel Martijn de Bruin, ${ }^{3}$ \\ Chantal M A M van der Horst ${ }^{1}$
}

To cite: Horbach SER, Wolkerstorfer A, de Bruin DM, et al. Electrosclerotherapy for capillary malformations: study protocol for a randomised within-patient controlled pilot trial. BMJ Open 2017;7:e016401. doi:10.1136/ bmjopen-2017-016401

- Prepublication history for this paper is available online. To view please visit the journal online (http://dx.doi.org/10. 1136/bmjopen-2017-016401).

Received 13 February 2017 Revised 24 April 2017 Accepted 20 June 2017

\section{CrossMark}

${ }^{1}$ Department of Plastic, Reconstructive and Hand Surgery, Academic Medical Center (AMC), University of Amsterdam, Amsterdam, NoordHolland, Netherlands ${ }^{2}$ Department of Dermatology, Academic Medical Center (AMC), University of Amsterdam, Amsterdam, Noord-Holland, Netherlands

${ }^{3}$ Department of Biomedical Engineering and Physics, Academic Medical Center, University of Amsterdam, Amsterdam, Noord-Holland, Netherlands

Correspondence to Dr Sophie E R Horbach; s.e.horbach@amc.uva.nl

\section{ABSTRACT}

Introduction The current state-of-the-art treatment modality for hypertrophic capillary malformations (CMs), laser therapy, has a considerable rate of non-responders and recurrence. Intralesional bleomycin injections (or 'sclerotherapy') are commonly used to treat venous and Iymphatic malformations with an excellent effect, but these intravascular injections are not possible in CMs due to the small diameter of the vessels. Electroporation-an electric field applied to the tissue-could increase the permeability of endothelial cells, which could theoretically facilitate targeted localised bleomycin delivery. We therefore hypothesise that bleomycin injections in combination with electroporation-'electrosclerotherapy' (EST), also known as 'electrochemotherapy'—could potentially be a novel alternative treatment option for CMs. Methods and analysis In this randomised within-patient controlled pilot trial, 20 patients with hypertrophic CMs will be enrolled. Three regions of interest (ROIs) within the $\mathrm{CM}$ will be randomly allocated for treatment with $(A)$ EST, (B) bleomycin sclerotherapy without electroporation and (C) no treatment. Patients and outcome assessors are blinded for the treatment allocation. Treatment outcome for each ROI will be measured approximately 7 weeks after the treatment procedure, using patient-reported and physician-reported global assessment scores, colorimetry, laser speckle imaging and reporting of adverse events.

Ethics and dissemination The study protocol is approved by the ethics review committee of the Academic Medical Center, Amsterdam. Results will be published in peerreviewed medical journals and will be presented at international conferences and scientific meetings. Study results will be fed back to the patient population through website and social media notifications.

Trial registration number NCT02883023; Pre-results. NTR6169.

\section{INTRODUCTION}

Capillary malformations (CMs) are congenital abnormalities of the capillaries in the skin, also known as 'port-wine stains', which occur in approximately 3-5 of 1000 newborn. ${ }^{1-3}$ In these benign vascular birthmarks, ectatic dermal capillaries and postcapillary dermal
Strengths and limitations of this study

- This study has an innovative double blind (patient and outcome assessor), randomised and withinpatient controlled study design.

- During this study, we will gain experience with an interventional device that is used for this treatment indication for the first time.

- Since it concerns a feasibility study, it is not possible to statistically confirm safety or effectiveness in this study.

- There are no validated outcome measurement instruments available for measuring patientperceived treatment outcome for hypertrophic capillary malformations.

venules in the papillary and mid-reticular layers of the skin cause the characteristic red colour. According to the most recent classification of vascular anomalies of the International Society for the Study of Vascular Anomalies, CMs are categorised as 'vascular malformations', along with other types of congenital abnormally developed vessels: venous malformations, lymphatic malformations and arteriovenous malformations. ${ }^{4}$

While CMs are often light red and flat in young children, these birthmarks may progress into dark red or purple lesions with a nodular appearance with increasing age. ${ }^{56}$ The aetiology of CMs is unclear, but a lack of neural modulation of vascular flow is suspected. ${ }^{7}$ A few gene mutations have been associated with CMs. RASA1 gene mutations have been associated with the CM-arteriovenous malformation syndrome ${ }^{8}$ and the more recently discovered GNAQ mutation may be the main genetic determinant in isolated CMs. ${ }^{9}{ }^{10} \mathrm{CMs}$ can be associated with a wide range of syndromes, such a SturgeWeber syndrome in which facial CMs occur 
in combination with venous and CMs of the brain and eyes. ${ }^{11}$

Besides cosmetic burden, CMs can cause physical discomfort such as bleedings or compression of facial structures due to hypertrophy. The stigma of a disfiguring CM can cause significant psychological burden and a decreased quality of life. ${ }^{12} 13$

\section{Current treatment strategies}

In the last century, many treatment modalities have been used to treat CMs, such as argon laser, selective photothermolysis and epidermal cooling-all with disappointing results. ${ }^{14}$ In 1985, pulsed-dye laser (PDL) was introduced, and since then this therapy has been viewed as the stateof-the-art treatment modality for CMs. ${ }^{214}$ In laser therapy, abnormal blood vessels are targeted with photocoagulation while the overlying epidermis is spared. Some other less widely accepted treatment modalities are described in the literature, such as photodynamic therapy and intense pulsed light, but evidence suggests that these are inferior to the gold standard of laser therapy. ${ }^{2}$

Although PDL is the only widely accepted treatment option for CMs, treatment outcome is suboptimal in approximately $40 \%-50 \%$ of patients. ${ }^{15}$ Complete clearance is only seen in $~ 10 \%$ of patients. ${ }^{15}$ Furthermore, 10 years after laser therapy, redarkening of the CM occurred in $35 \%$ of cases. ${ }^{16}$ In CMs with hypertrophy, especially with early-onset, treatment response may be even poorer. ${ }^{17} 18$ In these cases, other lasers such as the alexandrite or long pulsed neodymium-doped yttrium aluminium garnet lasers may be somewhat more effective than PDL, ${ }^{1920}$ but hypertrophic CMs still remain a therapeutic challenge. Hence, there is a need for an alternative treatment option for CMs-especially for hypertrophic, treatment-resistant and recurrent CMs.

\section{Novel therapeutic intervention: electrosclerotherapy}

In this study, we hypothesise that intralesional bleomycin injections combined with electroporation ('electrosclerotherapy' (EST)) can be a feasible and safe alternative treatment option for CMs.

Intralesional bleomycin injections (sclerotherapy) are commonly used to treat vascular malformations of larger vessels (venous and lymphatic malformations). According to the literature, this treatment is effective in approximately $80 \%-90 \%$ of these patients. ${ }^{21}{ }^{22}$ Unfortunately, this therapy cannot be used in CMs because the diameter of capillary vessels is too small for a localised intravascular injection. ${ }^{23}$ Therefore, bleomycin cannot be delivered to the endothelial cells where it has its therapeutic sclerosing effect. ${ }^{24}$

'Electroporation' is a physical phenomenon that causes an alteration of the structure of cell membranes through the exposure of cells to a short but intense electric field.

This modification of the cell membrane increases its permeability and allows molecules that normally do not cross the cell membrane to be transported to the intracellular environment. ${ }^{25}$ Therefore, electroporation is an ideal method for localised drug delivery, in particular for localised bleomycin delivery, while avoiding systemic side effects.

EST is the combination of electroporation with a sclerosing drug. In this protocol, we will use the definition EST for electroporation combined with intralesional bleomycin injections. In EST, electroporation will facilitate the delivery of bleomycin to the endothelium of abnormally developed capillary vessels, causing a localised drug effect. We hypothesise that the sclerosing effect of bleomycin will reach the endothelium, where it initiates vascular destruction and ultimately leads to regression of the CM. In oncology, EST is also referred to as 'electrochemotherapy' because of the cytotoxic properties of bleomycin. EST is exactly the same treatment as electrochemotherapy, however, it is named differently because it aims to enhance the sclerosing effect of bleomycin rather than the cytotoxic effect. Electrochemotherapy is already used in clinical practice throughout the world, for a wide range of indications varying from keloids to Kaposi sarcoma. ${ }^{26-28}$

With a pilot study, we aim to explore if EST has the potential to become a new treatment modality for CMs. This pilot study will not be hypothesis testing, but will provide us preliminary information about the feasibility of this intervention and will identify modifications needed in the design of a larger, ensuing hypothesis testing study. If we find preliminary evidence of efficacy of EST, we will continue investigating if EST could be a valuable addition to the current treatment guidelines for CMs.

\section{Objectives}

Primary objective:

- To determine the feasibility of EST as a treatment option for CMs and the current study design.

Secondary objective(s):

- To explore the efficacy of EST as a treatment option for CMs.

- To explore the safety of EST as a treatment option for CMs.

\section{METHODS AND ANALYSIS}

\section{Trial design}

A prospective, randomised, within-patient controlled, phase II, pilot study. This is a monocentre study in the Academic Medical Center (AMC) in Amsterdam.

In this study, we will include patients with hypertrophic CMs. In each patient, we will randomly allocate three different regions within the hypertrophic CM to (A) EST, (B) bleomycin injections alone or (C) no treatment. Treatment outcome will be measured per treated region, approximately 7 weeks after the treatment procedure, using patient-reported and physician-reported global assessment scores, colorimetry, laser speckle imaging and reporting of adverse events. A simplified study flow chart is shown in figure 1. 


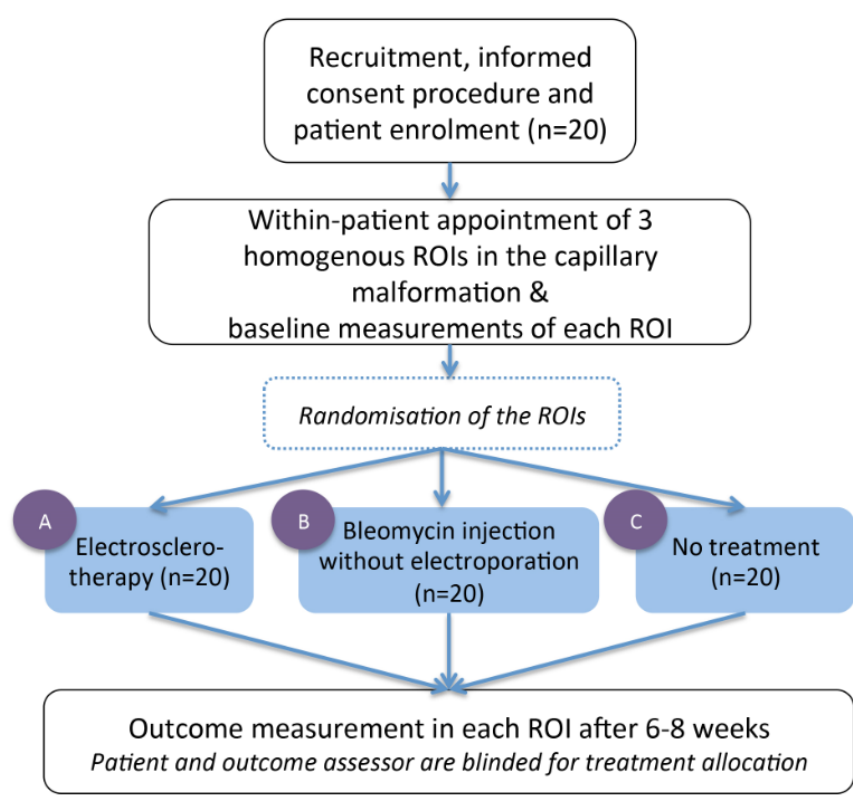

Figure 1 Study flow chart including randomisation procedure. ROI, region of interest.

\section{Participants and recruitment}

In this study, patients with hypertrophic CMs are included. Hypertrophy is defined as increased thickness or nodularity of the CM. This can be found in approximately $20 \%$ of CMs ${ }^{29}$ All inclusion and exclusion criteria are presented in table 1. Patients presenting at the Netherlands Institute of Pigment Disorders and at the outpatient clinics of the Dermatology and Plastic and Reconstructive Surgery departments of the AMC in Amsterdam and members of Dutch organisations and support groups for patients with CMs will be invited to participate. Patients will be contacted by telephone, mail, email or social media to inform them about the study. Patients expressing interest in participating will be assessed for eligibility by the investigators. When a patient is eligible for participation, detailed study information will be provided both verbally and in writing. If the patient chooses to participate, the informed consent form has to be signed, witnessed by a member of the research group.

\section{Proposed sample size}

Since this pilot study has an explorative character, one of the main objectives is to gain information for conducting a larger scale therapeutic effectiveness trial. Therefore, no formal power or sample size calculation was performed. This pilot study is not hypothesis testing and no inferential statistical analyses will be proposed.

To explore the feasibility, efficacy and safety of EST, we aim to include approximately 20 patients. This number was determined by our research group based on the necessities for examining feasibility (eg, learning curve of the treating physician), our current patient flow and budgetary constraints.

\section{Randomisation, treatment allocation and blinding}

Three regions of interest (ROIs) of approximately $1.5 \times 1.5 \mathrm{~cm}$ with homogeneous appearance (based on hypertrophy/nodularity and colour) within the $\mathrm{CM}$ will be appointed by the investigator. These regions will be numbered ROI-1, ROI-2 and ROI-3. An example of this procedure is shown in figure 2. These ROIs will be randomly allocated to (A) treatment with EST,

Table 1 Inclusion and exclusion criteria

\begin{tabular}{|c|c|}
\hline Inclusion criteria & Exclusion criteria \\
\hline
\end{tabular}




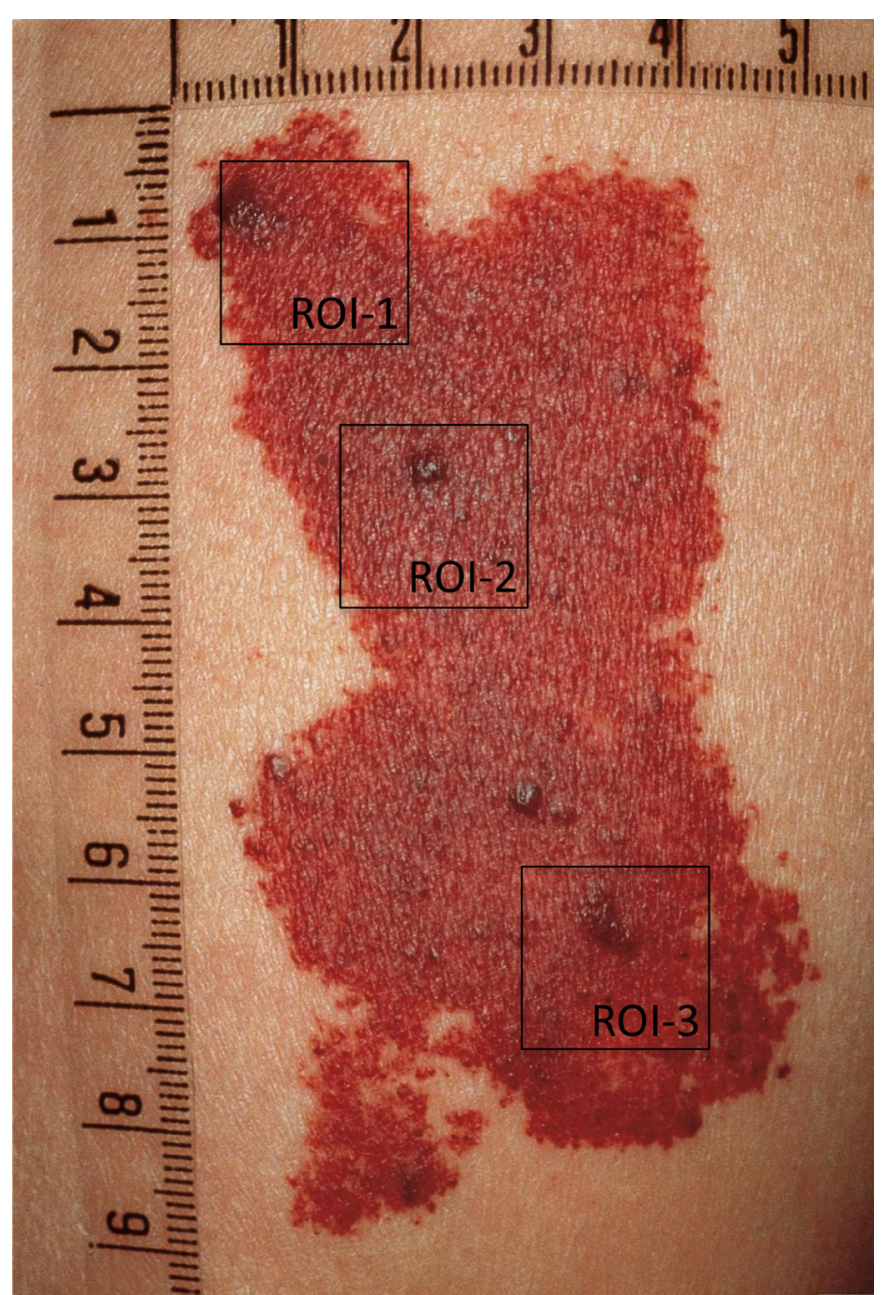

Figure 2 Example of the selection and numbering of three homogeneous regions of interest (ROIs) in the capillary malformation (image source: https://www.enzyklopaediedermatologie.de/gefaessmedizin/naevus-flammeusubersicht-2634\#\&gid=1\&pid=14).

(B) intralesional bleomycin injection or (C) no treatment. The goal of randomisation is to ensure that the treating physician does not choose a less severe region for the study intervention, compared with the control interventions. The randomisation will be based on a digitally generated list, which was created by a randomisation specialist using ALEA software. The combination of numbers and letters (eg, 1B-2A-3C) will appear in random order for each patient.

The patient will not be informed about the randomisation results. We will take measures to blind the patient for treatment allocation of the three ROIs. All ROIs will be anaesthetised and the patient will not be able to observe the treatment procedure. Only the ROIs receiving bleomycin and/or EST will be truly injected, in the ROI receiving no treatment the injections will be simulated with needle pricks. However, it may be difficult to completely blind the patient for the electroporation procedure, as the patient may feel the electric pulses near the ROI that is treated with EST. The treating physicians are not blinded as this is impossible in practice. The

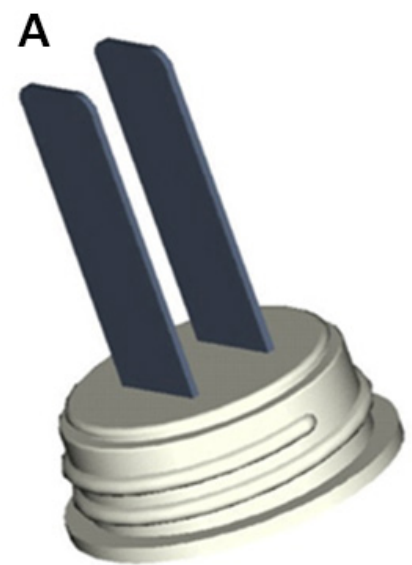

B

Figure 3 (A) Plate electrodes. (B) Hexagonal needle electrodes.

outcome assessor is an investigator who was not involved in the treatment procedure and he/she will be blinded for the treatment allocation of the ROIs.

\section{Interventions}

Participants will be treated in one treatment session. EST will be performed using the Cliniporator (IGEA, Carpi, Italy). The Cliniporator (model EPS02) is a CE-certified device for reversible electroporation of tissue. A handle with electrodes is connected to the device. The electrodes are placed on the tissue to generate an electric field. Two examples of electrodes are depicted in figure 3 . The plate electrodes are preferred in this study, as they do not penetrate the skin and are therefore generally less harmful. There is, however, an option to switch to needle electrodes for deeper lesions. In the ROI receiving EST, electroporation of the tissue is combined with intralesional bleomycin injections. The dosage of bleomycin is $0.25 \mathrm{mg}$ or units $/ \mathrm{cm}^{3}$, based on the standard operating procedures for electrochemotherapy, as previously described by Mir et $a l^{30}$ One milligram potency of bleomycin corresponds with approximately 1 USP, 1 unit and 1000 international units (IU), the latter is the most widely accepted measure internationally. ${ }^{31}$ The second ROI will be treated with bleomycin injections following the same protocol, but electroporation will not be applied. The last ROI will not be treated. Measurements will only be performed on these three ROIs. The remaining area of the CM, outside of the ROIs, will not be treated and will not be measured. Since the safety profile of EST is not yet clearly defined, we will not treat the entire CM with EST. In the future, when there is more evidence about the safety and effectiveness of EST in hypertrophic CMs, this therapy may become available for treating entire CMs.

\section{Primary outcome measures}

Currently there is no objective outcome measure for hypertrophic CMs, which measures both the hypertrophy and the colour of the CM. Therefore, we chose the (change in) appearance measured by both the observer and the patient as the primary outcome measure in this 
study. This is measured using global assessment of change scores and the Patient and Observer Scar Assessment Scale (POSAS) V.2.0.

The global assessment of improvement after treatment is estimated by the physician and the patient separately. This global assessment score is a subjective measure which is only assessed after treatment by the patient and the outcome assessor. Global improvement is categorised in five groups: no improvement or worsening $(0 \%$ regression), minor improvement ( $>0 \%-25 \%$ regression), moderate improvement ( $>25 \%-50 \%$ regression), marked improvement ( $>50 \%-75 \%$ regression) and near complete or complete remission ( $>75 \%-100 \%$ regression). Both the patient and the physician choose a category which expresses the amount of regression best.

The POSAS is a reliable and validated subjective assessment scale that consists of two numeric scales: the Patient Scar Assessment Scale (patient scale) and the Observer Scar Assessment Scale (observer scale). ${ }^{33}$ The observer scale comprises six parameters: vascularisation, pigmentation, thickness, relief, pliability and surface area. The six parameters of the patient scale are: pain, itching, colour, stiffness, thickness and surface irregularity. All items are scored in relation to the normal skin before and after treatment on a Likert scale from 1 to 10. Since the POSAS scale was initially developed for scars, bleeding symptoms of CMs were not included in the questionnaire. We therefore added one question to the POSAS scale: 'Did bleeding of the CM burden you in the last weeks?'.

\section{Secondary outcome measures \\ Adverse events}

All adverse events will be reported during follow-up. These will be classified according to the Common Terminology Criteria for Adverse Events V.4.0 guidelines.

\section{Colorimetry}

The difference in colour of the ROI in the $\mathrm{CM}$, in relation to the colour of the contralateral normal skin, will be measured using colorimetry (Minolta Chroma Meter model CR-300). The Minolta Chroma Meter uses the $\mathrm{L}^{*} \mathrm{a} * \mathrm{~b} *$ colour coordinate system, where $\mathrm{L}^{*}$ denotes lightness, $a^{*}$ values from green to red and $b^{*}$ values from blue to yellow. The difference in colour between the ROI and the normal skin will be calculated using the $\mathrm{L}^{*} \mathrm{a} * \mathrm{~b} *$ coordinates and is denoted by $\Delta \mathrm{E}$. The $\Delta \mathrm{E}$ will be determined before and after the treatment according to the following equation (CIE76-formula): $\Delta \mathrm{E}=\left((\Delta \mathrm{L})^{2}+(\Delta \mathrm{a})^{2}+(\Delta \mathrm{b})^{2}\right)^{1 / 2}$.

A low value for $\Delta \mathrm{E}$ indicates a small colour difference or a light $\mathrm{CM}$, and a high value for $\Delta \mathrm{E}$ represents a large colour difference.

\section{Laser speckle contrast imaging}

Laser speckle contrast imaging (LSCI) is an optical imaging technique and will be used to visualise perfusion in the ROIs of the CMs in this study. Perfusion will be measured in perfusion units per second.

\section{Feasibility outcome measures}

We will report on feasibility aspects, among others the feasibility of the intervention technique, applicability of the POSAS score in this CM study, the randomisation technique and the blinding procedure. We will register any problems occurring in these fields through interviewing with patients, physicians and investigators. Furthermore, recruitment rates and consent rates will be reported.

\section{Study procedures}

Visit 1

After the patient has signed the informed consent form, patient characteristics (gender, age, previous therapies, location of $\mathrm{CM}$ and patient-reported symptoms) will be collected. Based on hypertrophy, nodularity and colour, the investigator will select three homogeneous ROIs as described in the randomisation procedure. Lesions in the face, in the skin overlying joints or in mucosal tissue will not be treated. The contour of the CM and the three ROIs will be drawn onto a transparent foil including ROI-numbers. The CM and the three ROIs will be photographed. The patient and the physician will complete the POSAS score for each of the three ROIs. Furthermore, the colour and perfusion of the three ROIs will be measured using colorimetry LSCI, respectively.

After the baseline measurements, the ROIs will be randomised to either (A) EST, (B) bleomycin injections alone or $(\mathrm{C})$ no treatment. The ROIs to be treated will be anaesthetised using a nerve block with lidocaine $2 \%$. Afterwards, the ROIs will be treated with injections of bleomycin $\left(0.25 \mathrm{mg}\right.$ or units $\left./ \mathrm{cm}^{3}\right)$, the ROI that was randomised to EST will be treated with electric pulses using the Cliniporator within $10 \mathrm{~min}$ after the bleomycin injection. The treatment procedures will take place in the outpatient clinic.

\section{Telephone interview}

After 24-48hours, the investigator will call the patient to check for pain, swelling, oozing an wound healing. If there are alarming symptoms, the patient will be invited to come to the outpatient clinic.

\section{Visit 2}

After 1 week ( \pm 3 days), the patient will visit the outpatient clinic for a wound check. The ROIs will be photographed and any adverse events will be registered in the electronic patient file and the research dossier.

\section{Visit 3}

At visit 3 after $7 \pm 1$ weeks, the primary and secondary outcome measures will be measured (global assessment +POSAS, LSCI and colorimetry) following the same procedure as the baseline measurements of visit 1 . Adverse events will be registered. During the last visit, the patient and the physician will discuss if the patient has further treatment needs. A schematic overview of all visits is shown in table 2. 
Table 2 Schematic overview of study procedures and measurements for each study visit

\begin{tabular}{|c|c|c|c|c|}
\hline & \multicolumn{4}{|c|}{ Visits (t=time after intervention) } \\
\hline & $\begin{array}{c}\text { Visit 1 } \\
\text { (intake), } t=0\end{array}$ & $\begin{array}{c}\text { Telephone interview on } \\
\text { crusting, oozing, vesicles, } \\
t=24-48 \text { hours }\end{array}$ & $\begin{array}{c}\text { Visit } 2 \\
\text { (wound check), } \\
t=1 \text { week } \pm 3 \text { days }\end{array}$ & $\begin{array}{c}\text { Visit } 3 \\
\text { (end of study), } \\
t=7 \pm 1 \text { week }\end{array}$ \\
\hline Informed consent & $x$ & & & \\
\hline Standardised photography & $x$ & & $x$ & $\mathrm{x}$ \\
\hline Patient's global assessment (POSAS) & $x$ & & & $x$ \\
\hline Physician's global assessment (POSAS) & $x$ & & & $x$ \\
\hline $\mathrm{LSCl}$ & $x$ & & & $x$ \\
\hline Colorimetry & $\mathrm{x}$ & & & $x$ \\
\hline Randomisation of ROls & $x$ & & & \\
\hline $\begin{array}{l}\text { Intervention (EST, only bleomycin } \\
\text { injection or no treatment) of the three } \\
\text { ROls }\end{array}$ & $x$ & & & \\
\hline Registration of adverse events & & $\mathrm{x}$ & $\mathrm{x}$ & $\mathrm{x}$ \\
\hline
\end{tabular}

EST, electrosclerotherapy; LSCI, laser speckle contrast imaging; POSAS, Patient and Observer Scar Assessment Scale; ROI, region of interest.

\section{Trial organisation and monitoring}

Good Clinical Practice (GCP) approved software will be used for database management. An independent and qualified data monitor will provide GCP data monitoring.

\section{Data analysis plan}

Data will be analysed per protocol. Since this study has an explorative character, primarily focusing on the feasibility of the study design and procedures, we will mainly use descriptive statistics in combination with qualitative data. Categorical data will be expressed as numbers (n) and percentages (\%), continuous variables will be presented with a mean and SD (normally distributed data) or with a median and IQRs (non-parametrical data).

For an exploratory comparison of the treatment allocation groups and within-patient outcomes pretreatment and post-treatment, we will also use (non-parametric) statistics, tailored to the paired structure of the data. However, we will interpret these results with caution, since we realise that our study does not have sufficient power to formally test efficacy hypotheses. Statistical uncertainty will be expressed in $95 \%$ CIs.

\section{ETHICS AND DISSEMINATION Confidentiality}

All data are handled confidentially. Only the investigators as listed in this protocol have access to the source data. No identifiable information will be entered in the database. A subject identification code list is used to link the data in the database to the subject. The subject identification code is not based on patient initials or birth date. The key to the code is safeguarded by the investigator (SERH) in a password encrypted file.
The handling of personal data in this study is in compliance with the Dutch Personal Data Protection Act (in Dutch: De Wet Bescherming Persoonsgegevens).

\section{Data Safety Monitoring Board}

A Data Safety Monitoring Board (DSMB) is established to perform ongoing safety surveillance and to perform interim analyses on the safety data. This is an independent committee consisting of one urologist and one vascular surgeon, familiar with the research topic and one statistician/epidemiologist. The members of this DSMB are not involved in the present study and have no conflict of interest with the sponsor of the study. In short, the task and responsibility of the DSMB are to monitor safety data when $50 \%$ of the patients $(n=10)$ have been randomised, and, if required to monitor safety on ad hoc basis. Any mortality will be reported directly to the DSMB and evaluated for cause of death and possible trial-related (serious) adverse events. The justification for recommendation to terminate the study to clear harm will be based on a notably increase of (serious) adverse events, with special attention to the occurrence of severe local events (skin necrosis or severe ulceration, nerve damage, severe bleedings or any other complication requiring surgical intervention). Statistically stopping boundaries will not be prespecified. At the time of interim analysis, the DSMB will also evaluate the overall conduct of the trial in terms of protocol compliance, recruitment, losses to follow-up, completeness of the data and reports of the independent data monitor.

\section{Dissemination}

Study results will be published in a peer-reviewed journal and will be presented at international conferences. Furthermore, study results will be fed back to the patient 
population via news feeds on websites and social media. This research was not funded by grants from funding agencies in the public, commercial or not-for-profit sectors.

\section{DISCUSSION}

In this pilot study, we will explore the potential of EST as a new treatment modality for CMs. A new treatment modality would benefit many patients with CMs, especially those with hypertrophic, treatment-resistant and recurrent $\mathrm{CMs}$, for whom conventional laser therapy is not feasible. This pilot study is the essential first step in the quest of finding an alternative treatment option for these patients. Although EST has never been used to treat hypertrophic CMs before, there is preclinical and clinical evidence of its working mechanism and therapeutic effectiveness in other skin lesions.

The working mechanism of EST has been appointed to the synergistic effect of electroporation and bleomycin. The local effect of bleomycin is enhanced because electroporation has the potential to increase the concentration of low permeable drugs within the cell-up to 10000 times for bleomycin. ${ }^{25} 26$ Multiple preclinical studies have shown that the effect of EST on nodular skin lesions is largely due to a vascular disrupting effect on the supplying blood vessels of the lesion. ${ }^{34-36}$ In a preclinical study of Sersa $e t a l^{37}$ on murine fibrosarcoma cells, it was found that electrochemotherapy led to an immediate vascular disrupting effect of tumour blood flow reduction, hypoxia and decreased tumour oxygenation. Furthermore, endothelial cells of tumour blood vessels seemed to be exposed to a $\sim 40 \%$ higher electric field than the surrounding tumour cells. The subsequently occurring endothelial swelling and apoptosis led to a delayed vascular disrupting effect of tumour necrosis, a prolonged reduction of tumour blood flow and finally tumour regression. This phenomenon (vascular destruction, reduction of blood flow and lesion regression) is also the desired effect of treatment modalities for CMs.

Clinically, EST has already been used in many skin lesions, varying from melanoma to basal cell carcinoma, with objective response rates ranging from $69.7 \%$ to $100 \% .^{38}$ It has recently been suggested as the new 'standard of care' for Kaposi sarcomas, tumours with a predominant vascular origin, since $75 \%$ of patients had complete lesion clearance after only one treatment procedure. ${ }^{26}$ In a study of Manca et $a t^{28}$ in which 20 patients with 35 keloids and hypertrophic scars were treated with EST, scar height, scar volume, erythema and pliability of the scars improved significantly. The present pilot study will have to determine if EST is also effective in reducing hypertrophy and erythema in CMs.

No serious adverse events have been reported in the literature on EST for other cutaneous or subcutaneous lesions. The most frequently reported complications are transient pain, erythema or other local skin toxicity, hyperpigmentation and fever. ${ }^{26}{ }^{39}$ In a review of Testori et $a l,{ }^{40}$ authors mention that treated lesions heal without a scar, due to the fact that EST does not denature collagenic extracellular matrix and proteins, unlike other physical ablation technologies. In this feasibility trial, the safety and effectiveness of EST as a treatment option for hypertrophic CMs will be explored. When the results of this study provide preliminary evidence for the safety and effectiveness of EST in hypertrophic CMs, a larger ensuing hypothesis testing trial will be conducted.

Acknowledgements The authors are grateful to Rob de Haan, Michal Heger and Sanne Jansen for their valuable comments on the study protocol.

Contributors SERH, AW and CMAMH were involved in the inception of the study. SERH, AW, DMB and CMAMH conceptualised and designed the study. SERH, AW and CMAMH will collect data. SERH, AW, DMB and CMAMH will perform data analyses or interpretation of data. SERH drafted the initial manuscript of this study protocol. AW, DMB and CMAMH revised the manuscript for intellectual content and approved the final manuscript as submitted.

Funding This work was supported by a personal academic research scholarship, received by the corresponding author SERH. IGEA S.p.A. provided the materials needed for this study free of charge.

Competing interests None declared.

Ethics approval The Institutional Review Board of the Academic Medical Center in Amsterdam approved the study protocol (V1.1, 25 October 2016).

Provenance and peer review Not commissioned; externally peer reviewed.

Open Access This is an Open Access article distributed in accordance with the Creative Commons Attribution Non Commercial (CC BY-NC 4.0) license, which permits others to distribute, remix, adapt, build upon this work non-commercially, and license their derivative works on different terms, provided the original work is properly cited and the use is non-commercial. See: http://creativecommons.org/ licenses/by-nc/4.0/

(c) Article author(s) (or their employer(s) unless otherwise stated in the text of the article) 2017. All rights reserved. No commercial use is permitted unless otherwise expressly granted.

\section{REFERENCES}

1. Jacobs $A H$, Walton $R G$. The incidence of birthmarks in the neonate. Pediatrics 1976;58:218-22.

2. Chen JK, Ghasri P, Aguilar G, et al. An overview of clinical and experimental treatment modalities for port wine stains. J Am Acad Dermatol 2012;67:289-304.

3. Kanada KN, Merin MR, Munden A, et al. A prospective study of cutaneous findings in newborns in the United States: correlation with race, ethnicity, and gestational status using updated classification and nomenclature. J Pediatr 2012;161:240-5.

4. Wassef M, Blei F, Adams D, et al. Vascular anomalies classification: recommendations from the international society for the study of vascular anomalies. Pediatrics 2015;136:e203-e214.

5. Barsky SH, Rosen S, Geer DE, et al. The nature and evolution of port wine stains: a computer-assisted study. J Invest Dermatol 1980;74:154-7.

6. Lee JW, Chung HY, Cerrati EW, et al. The Natural history of soft tissue hypertrophy, bony hypertrophy, and nodule formation in patients with untreated head and neck capillary malformations. Dermatol Surg 2015;41:1241-5.

7. Smoller BR, Rosen S. Port-wine stains. A disease of altered neural modulation of blood vessels? Arch Dermatol 1986;122:177-9.

8. Revencu N, Boon LM, Mendola A, et al. RASA1 mutations and associated phenotypes in 68 families with capillary malformationarteriovenous malformation. Hum Mutat 2013;34:1632-41.

9. Tan W, Nadora DM, Gao L, et al. The somatic GNAQ mutation (R183Q) is primarily located within the blood vessels of port wine stains. J Am Acad Dermatol 2016;74:380-3.

10. Frigerio A, Wright K, Wooderchak-Donahue W, et al. Genetic Variants Associated with Port-Wine Stains. PLoS One 2015;10:e0133158.

11. Nozaki T, Nosaka S, Miyazaki O, et al. Syndromes associated with vascular tumors and malformations: a pictorial review. Radiographics 2013;33:175-95. 
12. Lanigan SW, Cotterill JA. Psychological disabilities amongst patients with port wine stains. Br J Dermatol 1989;121:209-15.

13. Masnari O, Schiestl C, Rössler J, et al. Stigmatization predicts psychological adjustment and quality of life in children and adolescents with a facial difference. $J$ Pediatr Psychol 2013;38:162-72.

14. Kelly KM, Choi B, McFarlane S, et al. Description and analysis of treatments for port-wine stain birthmarks. Arch Facial Plast Surg 2005;7:287-94.

15. Koster PH, Bossuyt PM, van der Horst CM, et al. Assessment of clinical outcome after flashlamp pumped pulsed dye laser treatment of portwine stains: a comprehensive questionnaire. Plast Reconstr Surg 1998;102:42-8.

16. Huikeshoven $\mathrm{M}$, Koster $\mathrm{PH}$, de Borgie $\mathrm{CA}$, et al. Redarkening of portwine stains 10 years after pulsed-dye-laser treatment. N Engl J Med 2007;356:1235-40.

17. Prather HB, Arndt KA. The Development of hypertrophy in portwine stains, a common phenomenon that affects treatment recommendations. Dermatol Surg 2015;41:1246-8.

18. Passeron T, Salhi A, Mazer JM, et al. Prognosis and response to laser treatment of early-onset hypertrophic port-wine stains (PWS). J Am Acad Dermatol 2016;75:64-8.

19. van Drooge AM, Bosveld B, van der Veen JP, et al. Long-pulsed 1064 $\mathrm{nm}$ Nd:YAG laser improves hypertrophic port-wine stains. J Eur Acad Dermatol Venereol 2013;27:1381-6.

20. Tierney EP, Hanke CW. Alexandrite laser for the treatment of port wine stains refractory to pulsed dye laser. Dermatol Surg 2011;37:1268-78.

21. Horbach SE, Lokhorst MM, Saeed P, et al. Sclerotherapy for low-flow vascular malformations of the head and neck: A systematic review of sclerosing agents. J Plast Reconstr Aesthet Surg 2016;69:295-304

22. Horbach SE, Rigter IM, Smitt JH, et al. Intralesional bleomycin injections for vascular malformations: a systematic review and metaanalysis. Plast Reconstr Surg 2016;137:244-56.

23. Muir T, Kirsten M, Fourie P, et al. Intralesional bleomycin injection (IBI) treatment for haemangiomas and congenital vascular malformations. Pediatr Surg Int 2004;19:766-73.

24. Sato D, Kurita M, Ozaki M, et al. Extravascular injection of sclerotic agents does not affect vessels in the rat: experimental implications for percutaneous sclerotherapy of arteriovenous malformations. Eur $J$ Vasc Endovasc Surg 2012;44:73-6.

25. Giardino R, Fini M, Bonazzi V, et al. Electrochemotherapy a novel approach to the treatment of metastatic nodules on the skin and subcutaneous tissues. Biomed Pharmacother 2006;60:458-62.

26. Di Monta G, Caracò C, Benedetto L, et al. Electrochemotherapy as "new standard of care" treatment for cutaneous Kaposi's sarcoma. Eur J Surg Oncol 2014;40:61-6.
27. Guida M, Campana LG, Curatolo P, et al. Local treatment with electrochemotherapy of superficial angiosarcomas: Efficacy and safety results from a multi-institutional retrospective study. J Surg Oncol 2016;114:246-53.

28. Manca G, Pandolfi P, Gregorelli C, et al. Treatment of keloids and hypertrophic scars with bleomycin and electroporation. Plast Reconstr Surg 2013;132:621e-30.

29. van Drooge AM, Beek JF, van der Veen JP, et al. Hypertrophy in portwine stains: prevalence and patient characteristics in a large patient cohort. J Am Acad Dermatol 2012;67:1214-9.

30. Mir LM, Gehl J, Sersa G, et al. Standard operating procedures of the electrochemotherapy: Instructions for the use of bleomycin or cisplatin administered either systemically or locally and electric pulses delivered by the CliniporatorTM by means of invasive or non-invasive electrodes. European Journal of Cancer Supplements 2006;4:14-25.

31. Stefanou A, Siderov J. Society of Hospital Pharmacists of Australia Committee of Specialty Practice in Oncology. Medical errors. Dosage nomenclature of bleomycin needs to be standardised to avoid errors. BMJ 2001;322:1423-4.

32. Stefanou A. Not again. BMJ 2001;322:548.

33. van der Wal MB, Tuinebreijer WE, Lundgren-Nilsson $\AA$, et al. Differential item functioning in the Observer Scale of the POSAS for different scar types. Qual Life Res 2014;23:2037-45.

34. Markelc B, Sersa G, Cemazar M. Differential mechanisms associated with vascular disrupting action of electrochemotherapy: intravital microscopy on the level of single normal and tumor blood vessels. PLoS One 2013;8:e59557.

35. Cemazar M, Parkins CS, Holder AL, et al. Electroporation of human microvascular endothelial cells: evidence for an anti-vascular mechanism of electrochemotherapy. Br J Cancer 2001;84:565-70.

36. Sersa G, Cemazar M, Miklavcic D, et al. Tumor blood flow modifying effect of electrochemotherapy with bleomycin. Anticancer Res 1999;19:4017-22.

37. Sersa G, Jarm T, Kotnik T, et al. Vascular disrupting action of electroporation and electrochemotherapy with bleomycin in murine sarcoma. Br J Cancer 2008;98:388-98.

38. Mali B, Jarm T, Snoj M, et al. Antitumor effectiveness of electrochemotherapy: a systematic review and meta-analysis. Eur J Surg Oncol 2013;39:4-16.

39. Solari N, Spagnolo F, Ponte E, et al. Electrochemotherapy for the management of cutaneous and subcutaneous metastasis: a series of 39 patients treated with palliative intent. J Surg Oncol 2014;109:270-4.

40. Testori A, Tosti G, Martinoli C, et al. Electrochemotherapy for cutaneous and subcutaneous tumor lesions: a novel therapeutic approach. Dermatol Ther 2010;23:651-61. 
Correction: Electrosclerotherapy for capillary malformations: study protocol for a randomised within-patient controlled pilot trial

Horbach SER, Wolkerstorfer A, de Bruin DM, et al. Electrosclerotherapy for capillary malformations: study protocol for a randomised within-patient controlled pilot trial. BMJ Open 2017;7:e016401. doi: 10.1136/bmjopen-2017-016401

'Sanne M Jansen' was missed off the original author list. The correct author list and affiliations should read:

Sophie E R Horbach, ${ }^{1}$ Albert Wolkerstorfer, ${ }^{2}$ Daniel Martijn de Bruin, ${ }^{3}$ Sanne M Jansen, ${ }^{3}$ Chantal M A M van der Horst ${ }^{1}$

1. Department of Plastic, Reconstructive and Hand Surgery, Academic Medical Center (AMC), University of Amsterdam, Amsterdam, Noord-Holland, Netherlands

2. Department of Dermatology, Academic Medical Center (AMC), University of Amsterdam, Amsterdam, Noord-Holland, Netherlands

3. Department of Biomedical Engineering and Physics, Academic Medical Center, University of Amsterdam, Amsterdam, Noord-Holland, Netherlands

Open Access This is an Open Access article distributed in accordance with the Creative Commons Attribution Non Commercial (CC BY-NC 4.0) license, which permits others to distribute, remix, adapt, build upon this work non-commercially, and license their derivative works on different terms, provided the original work is properly cited and the use is non-commercial. See: http://creativecommons.org/licenses/by-nc/4.0/

(C) Article author(s) (or their employer(s) unless otherwise stated in the text of the article) 2018. All rights reserved. No commercial use is permitted unless otherwise expressly granted.

BMJ Open 2018;8:e016401corr1. doi:10.1136/bmjopen-2017-016401corr1

Check for updates 